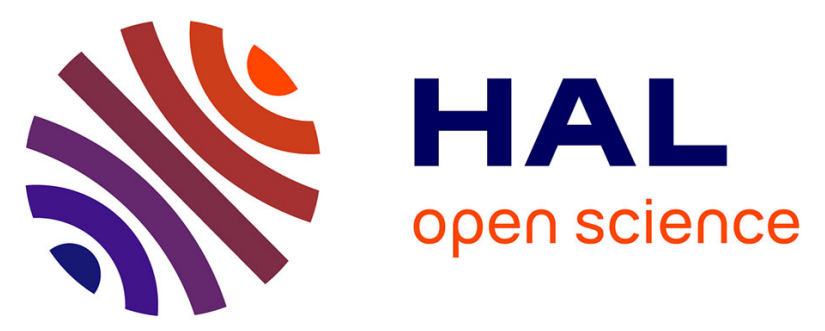

\title{
Multiscale identification of the random elasticity field at mesoscale of a heterogeneous microstructure using multiscale experimental observations
}

Manh-Tu Nguyen, Christophe Desceliers, Christian Soize, Jean-Marc Allain, H. Gharbi

\section{To cite this version:}

Manh-Tu Nguyen, Christophe Desceliers, Christian Soize, Jean-Marc Allain, H. Gharbi. Multiscale identification of the random elasticity field at mesoscale of a heterogeneous microstructure using multiscale experimental observations. International Journal for Multiscale Computational Engineering, 2015, 13 (4), pp.281-295. 10.1615/IntJMultCompEng.2015011435 . hal-01162147

\author{
HAL Id: hal-01162147 \\ https://hal.science/hal-01162147
}

Submitted on 31 Aug 2015

HAL is a multi-disciplinary open access archive for the deposit and dissemination of scientific research documents, whether they are published or not. The documents may come from teaching and research institutions in France or abroad, or from public or private research centers.
L'archive ouverte pluridisciplinaire HAL, est destinée au dépôt et à la diffusion de documents scientifiques de niveau recherche, publiés ou non, émanant des établissements d'enseignement et de recherche français ou étrangers, des laboratoires publics ou privés. 


\title{
Multiscale identification of the random elasticity field at mesoscale of a heterogeneous microstructure using multiscale experimental observations ${ }^{\underline{t}}$
}

\author{
M. T. Nguyen ${ }^{\mathrm{a}}$, C. Desceliers ${ }^{* a}$, C. Soize ${ }^{\mathrm{a}}$, J. M. Allain ${ }^{\mathrm{b}}$, H. Gharbi ${ }^{\mathrm{b}}$ \\ ${ }^{a}$ Université Paris-Est, Laboratoire Modélisation et Simulation Multi-Echelle, MSME UMR 8208 \\ CNRS, 5 bd Descartes, 77454 Marne-La-Vallée, Cedex 2, France \\ ${ }^{b}$ Ecole Polytechnique, Laboratoire de Mécanique des Solides, 91128, Palaiseau cedex, France
}

\begin{abstract}
This paper deals with a multiscale statistical inverse method for performing the experimental identification of the elastic properties of materials at macroscale and at mesoscale within the framework of a heterogeneous microstructure which is modeled by a random elastic media. New methods are required for carrying out such multiscale identification using experimental measurements of the displacement fields carried out at macroscale and at mesoscale with only a single specimen submitted to a given external load at macroscale. In this paper, for a heterogeneous microstructure, a new identification method is presented and formulated within the framework of the three-dimensional linear elasticity. It permits the identification of the effective elasticity tensor at macroscale, and the identification of the tensorvalued random field, which models the apparent elasticity field at mesoscale. A validation is presented first with simulated experiments using a numerical model based on the hypothesis of 2D-plane stresses. Then, we present the results given by the proposed identification procedure for experimental measurements obtained by digital image correlation (DIC) on cortical bone
\end{abstract}

Key words: Multiscale identification, heterogeneous microstructure, random elasticity field, mesoscale, multiscale experiments

\footnotetext{
International Journal for Multiscale Computational Engineering, 2015, doi: 10.1615/IntJMultCompEng.2015011435.

* Corresponding author

Email address: christophe.desceliers@univ-paris-est.fr(C. Desceliers)
} 


\section{Introduction}

The inverse methods devoted to the experimental identification of the elastic properties of materials at the macroscale and/or mesoscale have been extensively studied. The experimental identification of microstructural morphology by image analysis began in the 1980s [35, 36, 37], and it has led to significant advances in the identification of mechanical properties. We refer the reader, for instance, to [16] for the identification of elastic moduli from displacement-force boundary measurements, to $[38,57,58,31,30,47,8,33,45,46,48,34]$ for works devoted to the displacement field measurements in micro and macromechanics using digital image correlation in $2 \mathrm{D}$ and $3 \mathrm{D}$, including software developments, to $[32,11]$ for aspects concerning multiscale full-field measurements, to [10] for aspects concerning measurement errors in digital image correlation technique, to [22, 23, 9] for inverse problems in elasticity and for the identification of elastic parameters by using displacement field measurements, to [13, 12, 3, 41, 2, 4] for identification methods of mechanical parameters from full-field measurements in linear and nonlinear elasticity, and to $[7,24]$ for stochastic aspect of random media using a moving-window technique.

Concerning the identification of stochastic models, the methodologies for statistical inverse problems in finite and infinite dimensions are numerous and have given rise to numerous studies and publications. These methods make extensive use of the formulations and the tools of the functional analysis for the boundary value problems as well as those of probability theory, including mathematical statistics (finite and infinite dimensional cases). Concerning the mathematical statistics, one can refer to $[40,49]$ and to $[15,59,55,39]$ for the general principles on the statistical inverse problems. Early works on the statistical inverse identification of stochastic fields for random elastic media, using partial and limited experimental data, have primarily be devoted to the identification of the hyperparameters of prior stochastic models (such as the spatial correlation scales and the level of statistical fluctuations) $[19,20,1,18,17,29]$, and then, methodologies have recently been proposed for the identification of general stochastic representations of random fields in high stochastic dimension [52, 53, 44, 43]. Those probabilistic/statistical methods are able to solve the statistical inverse problems related to the identification of prior stochastic models for the apparent elastic fields at mesoscale. Nevertheless, such experimental identification, which is carried out 
using measurements of the displacement fields at macroscale and mesoscale on a single specimen submitted to a given external load at macroscale, requires new methods for identifying the statistical mean value of the random apparent elasticity tensor at mesoscale and the other hyperparameters controlling its prior stochastic model as, for instance, the spatial correlation lengths and the hyperparameters allowing the statistical fluctuations of the stochastic field to be controlled.

The present paper is not devoted to the presentation of a new stochastic model for representing the random elasticity field of a heterogeneous microstructure at mesoscale. This work proposes a new methodology for identifying a prior stochastic model of such random elasticity field using multiscale experimental data performed on a single specimen. Taking into account the major difficulty of this challenging statistical inverse problem induced by the high stochastic dimension of the tensor-valued random field to be identified, a parameterized prior stochastic model can be used in order to obtain a well-posed statistical inverse problem. Taking into account the mathematical properties of the tensor-valued random elasticity field of the heterogeneous elastic microstructure at mesoscale, the model proposed in [50] has been used for validating the multiscale identification.

In this paper, an identification method and its validation are presented using multiscale measurements. A new multiscale statistical inverse method is introduced for a heterogeneous microstructure within the framework of the three-dimensional linear elasticity. This method permits both the identification of the effective elasticity tensor at macroscale and the identification of the tensor-valued random field that models the apparent elasticity field at mesoscale. The methodology to perform the experimental identification of both the mean part and the statistical fluctuations of a stochastic model of the elasticity field at mesoscale of a heterogeneous microstructure using experimental data for a single specimen, has not been proposed in the literature. It should be noted that the random elasticity field is not a real valued random field, but a tensor-valued random field for which the entries of the tensor cannot be identified separately (entry by entry) due to algebraic properties that relate the entries. If a digital image correlation method was only used at mesoscale, then the mean part of the stochastic model at mesoscale could not be identified. Conversely, if a digital image correlation method was only used at macroscale, then the statistical fluctuations of the stochastic model at mesoscale could not be identified. Since, by assumptions, only a single specimen is experimentally tested, a multiscale digital image correlation must simultaneously be 
used at mesoscale and at macroscale for the specimen submitted to a given load. Such experimental procedure has specially been implemented and validated (see [42]). Therefore, the complete statistical information (mean and fluctuations) of the random elastic field at mesoscale must be transferred to the macroscale in order to use the macroscale measurements for identifying the mean part. Such a transfer of information is carried out by using a stochastic homogenization. These considerations have led the authors to propose an innovative and new multiscale experimental identification procedure.

In the presented work, a fundamental mechanical property is used: there exists a Representative Volume Element (RVE) that allows for a separation of the mesoscale with the macroscale. In the context of homogenization in micromechanics of heterogeneous materials, it is proved (see for instance [51] and references included) that, if such a RVE exists, then there is no statistical fluctuations at macroscale (with a very high level of probability), which means that there exists a scale separation. By definition of a RVE, it is necessary that the random elasticity field at mesoscale be ergodic and consequently, must be homogeneous (stationary). For the class of materials considered in this work (cortical bone) this hypothesis is experimentally verified. The statistical fluctuations of the cortical bone at macroscale are negligible, and therefore, there exist a RVE, and thus the hypothesis use (homogeneous random field at mesoscale) is reasonable. In addition, it should be noted that, if such a hypothesis was not verified, and taking into account that only one specimen is tested, then the statistical inverse problem would be an ill-posed problem, because the statistical averaging could not be done, due to the lack of the ergodic property.

It is assumed that the experimental measurements of the displacement field are available at macroscale and at mesoscale. The prior stochastic model is a nonGaussian tensor-valued random field adapted to the algebraic properties of the 3D-elasticity field and to the corresponding stochastic elliptic boundary value problem. The hyperparameters of the prior stochastic model of the apparent elasticity random field at mesoscale, are its statistical mean value, its spatial correlation lengths and its level of statistical fluctuations. The identification of such a stochastic model at mesoscale requires the knowledge of the effective elasticity tensor at macroscale, as well as the measurements of the displacements field at the two scales, simultaneously, for one given specimen submitted to a given static external load (as explained before). 
The theory is presented for the three-dimensional case. A numerical validation is presented first for the 2D plane stresses using simulated experiments. Then, we present the results given by the proposed identification procedure for experimental measurements obtained on cortical bone.

In all this paper, the framework of the three-dimensional linear elasticity is used. Consequently, and for instance, the terminology "strain tensor" will be used instead of "linearized strain tensor", etc.

\section{Multiscale experimental configuration}

The specimen (whose microstructure is complex and heterogeneous at microscale) occupies a bounded macroscopic domain $\Omega^{\text {macro }}$ in $\mathbb{R}^{3}$ (the three-dimensional Euclidean space). A surface force field, $\mathbf{f}^{\text {macro }}$, is applied on a part $\Sigma^{\text {macro }}$ of the boundary $\partial \Omega^{\text {macro }}$ of $\Omega^{\text {macro }}$. The other part $\Gamma^{\text {macro }}$ of $\partial \Omega^{\text {macro }}$ is fixed such that there is no rigid body displacement. At macroscale, the measured displacement field in $\Omega^{\text {macro }}$ is denoted by $\mathbf{u}_{\exp }^{\text {macro }}$ and its associated tensor-valued strain field is denoted by $\varepsilon_{\exp }^{\text {macro }}$.

Let $\Omega^{\text {meso }}$ be a subdomain of the specimen at mesoscale. It is assumed that $\Omega^{\text {meso }}$ is a representative elementary volume (REV). Let $\partial \Omega^{\text {meso }}$ be the boundary of $\Omega^{\text {meso }}$. Let $\mathbf{u}_{\mathrm{exp}}^{\text {meso }}$ be the experimental measurement of the $\mathbb{R}^{3}$-valued displacement field in $\Omega^{\text {meso }}$ at mesoscale. The associated tensor-valued strain field is denoted by $\varepsilon_{\exp }^{\text {meso }}$. It is assumed that the experimental measurements of displacement field $\mathbf{u}_{\mathrm{exp}}^{\text {meso }}$ are obtained only for one subdomain $\Omega^{\text {meso }}$ related to one specimen. The volume average at mesoscale, $\S_{\exp }^{\text {meso }}$, of $\varepsilon_{\exp }^{\text {meso }}$ is introduced such that

$$
\underbrace{\operatorname{meso}}_{\exp }=\frac{1}{\left|\Omega^{\text {meso }}\right|} \int_{\Omega^{\text {meso }}} \AA_{\exp }^{\operatorname{meso}}(\mathbf{x}) d \mathbf{x},
$$

in which $\mathbf{x}$ is the generic point in $\mathbb{R}^{3}$ and where $d \mathbf{x}$ is the volume element. The level of statistical fluctuations (around the volume average $\mathbb{E}_{\exp }^{\text {meso }}$ ) of $\mathbb{E}_{\exp }^{\text {meso }}$ is estimated by the quantity $\delta_{\exp }^{\text {meso }}$ defined by

$$
\delta_{\exp }^{\text {meso }}=\frac{\sqrt{V_{\exp }^{\text {meso }}}}{\|\underbrace{\operatorname{meso}}_{\exp }\|_{F}},
$$

in which

$$
V_{\exp }^{\text {meso }}=\frac{1}{\left|\Omega^{\text {meso }}\right|} \int_{\Omega^{\text {meso }}}\left\|\varepsilon_{\exp }^{\text {meso }}(\mathbf{x})-\underline{\exp }_{F}^{\text {meso }}\right\|_{F}^{2} d \mathbf{x}
$$


and where $\|T\|_{F}$ is the Frobenius norm such that, for any second-order tensor $T=\left\{T_{i j}\right\}_{i j}$, one has

$$
\|T\|_{F}^{2}=\sum_{i=1}^{3} \sum_{j=1}^{3} T_{i j}^{2} .
$$

\section{Multiscale statistical inverse problem}

In a first subsection, numerical indicators used in the identification procedure are defined. In the second subsection, the identification procedure is define for the case of $2 \mathrm{D}$ plane stresses.

\subsection{Construction of indicators for the identification procedure}

At macroscale, a deterministic boundary value problem is introduced for a threedimensional linear elastic medium, which models the specimen in its experimental configuration (geometry, surface forces and Dirichlet conditions). At this macroscale, the constitutive equation involves a prior model for the fourth-order elasticity tensor $C^{\text {macro }}$ (a) that is parameterized by a vector a. For a three-dimensional anisotropic elastic material, a represents the 21 constants of the fourth-order elasticity tensor. The boundary value problem is formulated in displacement and the solution is denoted by $\mathbf{u}^{\text {macro }}$ (deterministic $\mathbb{R}^{3}$-valued displacement field at macroscale, which depends on a). The tensor-valued strain field associated with $\mathbf{u}^{\text {macro }}$ is denoted by $\varepsilon^{\text {macro }}$ and depends on a. Tensor $C^{\text {macro }}(\mathbf{a})$ is unknown and must experimentally be identified, which means that the parameter a must be identified using the measurements of the displacement field at macroscale. Consequently, a first numerical indicator $\mathcal{I}_{1}(\mathbf{a})$ is introduced in order to quantify the distance between $\varepsilon_{\exp }^{\text {macro }}$ and $\varepsilon^{\text {macro }}(\mathbf{a})$. For a fixed value of parameter a, this indicator is defined by

$$
\mathcal{I}_{1}(\mathbf{a})=\left\||| \varepsilon_{\exp }^{\text {macro }}-\varepsilon^{\text {macro }}(\mathbf{a})\right\| \|^{2},
$$

in which

$$
\left\|\varepsilon_{\exp }^{\text {macro }}-\varepsilon^{\text {macro }}(\mathbf{a})\right\|\left\|^{2}=\int_{\Omega^{\text {macro }}}\right\| \varepsilon_{\exp }^{\text {macro }}(\mathbf{x})-\varepsilon^{\text {macro }}(\mathbf{x} ; \mathbf{a}) \|_{F}^{2} d \mathbf{x} .
$$

At mesoscale, two additional numerical indicators, $\mathcal{I}_{2}(\mathbf{b})$ and $\mathcal{I}_{3}(\mathbf{a}, \mathbf{b})$, are constructed to identify the hyperparameter $\mathbf{b}$ involved in the prior tensor-valued random field, $\mathbf{C}^{\text {meso }}(\mathbf{b})$, which models the apparent elasticity random field that is considered as the restriction to subdomain $\Omega^{\text {meso }}$ of a statistically homogeneous 
random field $\left\{\mathbf{C}^{\text {meso }}(\mathbf{x} ; \mathbf{b}), \mathbf{x} \in \mathbb{R}^{3}\right\}$.

Concerning the construction of the numerical indicator $\mathcal{I}_{2}(\mathbf{b})$, a stochastic boundary value problem is introduced for a three-dimensional linear elastic random medium occupying subdomain $\Omega^{\text {meso }}$, and for which the apparent elasticity random field is $\mathbf{C}^{\text {meso }}(\mathbf{b})$. This stochastic boundary value problem is formulated in displacement, and the solution is the random displacement field denoted by $\mathbf{U}^{\text {meso }}$ ( $\mathbb{R}^{3}$-valued random field) with the Dirichlet condition $\mathbf{U}^{\text {meso }}=\mathbf{u}_{\exp }^{\text {meso }}$ on boundary $\partial \Omega^{\text {meso }}$. The tensor-valued random strain field associated with $\mathbf{U}^{\text {meso }}$ is denoted by $\varepsilon^{\text {meso }}$. For any given hyperparameter $\mathbf{b}$, the numerical indicator $\mathcal{I}_{2}(\mathbf{b})$ is defined by

$$
\mathcal{I}_{2}(\mathbf{b})=\int_{\Omega^{\text {meso }}}\left(\delta^{\text {meso }}(\mathbf{x} ; \mathbf{b})-\delta_{\exp }^{\text {meso }}\right)^{2} d \mathbf{x},
$$

in which $\delta_{\exp }^{\text {meso }}$ is defined by Eq. (2) and where $\delta^{\text {meso }}(\mathbf{x} ; \mathbf{b})$ is defined by

$$
\delta^{\text {meso }}(\mathbf{x} ; \mathbf{b})=\frac{\sqrt{V^{\text {meso }}(\mathbf{x} ; \mathbf{b})}}{\left\|\underline{\underline{\varepsilon}}^{\text {meso }}(\mathbf{b})\right\|_{F}},
$$

and where $\underline{£}^{\text {meso }}(\mathbf{b})$ and $V^{\text {meso }}(\mathbf{x} ; \mathbf{b})$ are such that

$$
\begin{gathered}
\underline{\varepsilon}^{\text {meso }}(\mathbf{b})=\frac{1}{\left|\Omega^{\text {meso }}\right|} \int_{\Omega^{\text {meso }}}{ }_{\varepsilon^{\text {meso }}}(\mathbf{x} ; \mathbf{b}) d \mathbf{x}, \\
V^{\text {meso }}(\mathbf{x} ; \mathbf{b})=E\left\{\|\|_{\varepsilon}^{\text {meso }}(\mathbf{x} ; \mathbf{b})-\underline{\varepsilon}^{\text {meso }}(\mathbf{b}) \|_{F}^{2}\right\},
\end{gathered}
$$

in which $E$ is the mathematical expectation. It should be noted that, for all $\mathbf{b}$, $\underline{\varepsilon}^{\text {meso }}(\mathbf{b})={ }_{\exp }^{\text {meso }}$. Finally, the numerical indicator $\mathcal{I}_{3}(\mathbf{a}, \mathbf{b})$ quantifies the distance between the elasticity tensor $C^{\text {macro }}(\mathbf{a})$ used in the deterministic boundary value problem at macroscale, and the effective tensor $\mathbf{C}^{\text {eff }}(\mathbf{b})$ calculated by homogenization of the mesoscale stochastic model in subdomain $\Omega^{\text {meso }}$ which is a REV. We then have

$$
\mathcal{I}_{3}(\mathbf{a}, \mathbf{b})=\left\|C^{\text {macro }}(\mathbf{a})-E\left\{\mathbf{C}^{\mathrm{eff}}(\mathbf{b})\right\}\right\|_{F}^{2} .
$$

The identification of parameters $\mathbf{a}$ and $\mathbf{b}$ that describe the stochastic model of the apparent elasticity random field $\mathbf{C}^{\text {meso }}(\mathbf{b})$ at mesoscale is obtained by solving a multi-objective optimization problem for the three indicators $\mathcal{I}_{1}(\mathbf{a}), \mathcal{I}_{2}(\mathbf{b})$, and $\mathcal{I}_{3}(\mathbf{a}, \mathbf{b})$.

\subsection{Identification procedure for $2 D$ plane stresses}

In this section, we present the particular case of $2 \mathrm{D}$ plane stresses, which will be the configuration of the experimental measurements presented in Section 5. 


\subsubsection{Prior stochastic model of the apparent elasticity random field for $2 D$ plane stresses}

In this subsection, a prior probabilistic model of the apparent elasticity random field is constructed for the case of 2D plane stresses. This prior probabilistic model will be used in the next sections (i) for the validation of the method in 2D plane stresses (Section 4), and (ii) for the application of the method with experimental measurements obtained through DIC method (Section 5).

At mesoscale, the prior stochastic model of the apparent elastic random field $\mathbf{C}^{\text {meso }}$ is indexed by subdomain $\Omega^{\text {meso }}$ which is assumed to be a REV. We are interested in using a stochastic representation of $\mathbf{C}^{\text {meso }}$ with a minimum of hyperparameters (dimension of vector $\mathbf{b}$ ), which is adapted to the elliptic boundary value problem corresponding to the linear elastostatic problem. Parametric stochastic models have been proposed for real-valued stochastic fields [24, 6, 5, 21], and for non-Gaussian tensor-valued random fields in the framework of the heterogeneous anisotropic linear elasticity [50, 51, 56, 54, 14], with important enhancements to take into account the material symmetry and the existence of elasticity bounds $[25,26,27,28]$. Hereinafter, the stochastic model for the apparent elastic tensorvalued random field $\mathbf{C}^{\text {meso }}$ is based on the model proposed in [50] for a heterogeneous anisotropic microstructure at the mesoscale.

In using the Voigt notation, for all $\mathbf{x}$ fixed in $\Omega^{\text {meso }}$, the random fourth-order elasticity tensor $\mathbf{C}^{\text {meso }}(\mathbf{x})$ can be represented by a $(6 \times 6)$ real random matrix. The strain random vector is then denoted by $\left(\varepsilon_{11}, \varepsilon_{22}, 2 \varepsilon_{12}, \varepsilon_{33}, 2 \varepsilon_{23}, 2 \varepsilon_{13}\right)$ and the associated random stress vector is denoted by $\left(\sigma_{11}, \sigma_{22}, \sigma_{12}, \sigma_{33}, \sigma_{23}, \sigma_{13}\right)$. Such numbering of those random vectors (that is not usual) has been chosen for the sake of simplicity in 2D plane stresses, for which the $(3 \times 3)$ compliance random matrix $\left[\mathbf{S}^{2 D}(\mathbf{x})\right]$ corresponds to the first $(3 \times 3)$ block of the $(6 \times 6)$ compliance random matrix $\left[\mathbf{S}^{\text {meso }}(\mathbf{x})\right]=\left[\mathbf{C}^{\text {meso }}(\mathbf{x})\right]^{-1}$.

The prior stochastic model of $\mathbf{C}^{\text {meso }}$ is then constructed in choosing $\left[\mathbf{S}^{\text {meso }}\right]=$ $\left\{\left[\mathbf{S}^{\text {meso }}(\mathbf{x})\right], \mathbf{x} \in \Omega^{\text {meso }}\right\}$ in the set $\mathrm{SFE}^{+}$(defined in $[50,51,54]$ ) of the nonGaussian second-order stochastic fields with values in the set of all the positivedefinite symmetric $(6 \times 6)$ real matrices denoted by $\mathbb{M}_{6}^{+}(\mathbb{R})$. For all $\mathbf{x}$ in $\Omega^{\text {meso }}$, the mean value is a given matrix $\left[\underline{S}^{\text {meso }}\right]=E\left\{\left[\mathbf{S}^{\text {meso }}(\mathbf{x})\right]\right\}$ in $\mathbb{M}_{6}^{+}(\mathbb{R})$, which is assumed independent of $\mathbf{x}$. The $\mathbb{M}_{6}^{+}(\mathbb{R})$-valued random field $\left\{\left[\mathbf{S}^{\text {meso }}(\mathbf{x})\right], \mathbf{x} \in \Omega^{\text {meso }}\right\}$ is parameterized by the symmetric matrix $\left[\underline{S}^{\text {meso }}\right]$, three spatial correlation lengths 
denoted by $\ell_{1}, \ell_{2}, \ell_{3}$, and one positive dispersion coefficient $\delta$ that controls the level of statistical fluctuations. Finally, an explicit generator of independent realizations (sample paths) of such a random field is completely defined in [50].

For the case of $2 \mathrm{D}$ plane stresses, for all $\mathbf{x}$ in $\Omega^{\text {meso }}$, the random matrix $\left[\mathbf{S}^{2 D}(\mathbf{x})\right]$ is defined as the left upper $(3 \times 3)$ block matrix of the random matrix $\left[\mathbf{S}^{\text {meso }}(\mathbf{x})\right]$. Consequently, the $\mathbb{M}_{3}^{+}(\mathbb{R})$-valued random field $\left\{\left[\mathbf{S}^{2 D}(\mathbf{x})\right], \mathbf{x} \in \Omega^{\text {meso }}\right\}$ is parameterized by the symmetric matrix $\left[\underline{S}^{2 D}\right]$ in $\mathbb{M}_{3}^{+}(\mathbb{R})$ (left upper $(3 \times 3)$ block matrix of $\left[\underline{S}^{\text {meso }}\right]$ ), one spatial correlation length $\ell=\ell_{1}=\ell_{2}$ and dispersion parameter $\delta$. The prior model of the apparent elasticity random field $\left[\mathbf{C}^{2 D}\right]=\left\{\left[\mathbf{C}^{2 D}(\mathbf{x})\right], \mathbf{x} \in \Omega^{\text {meso }}\right\}$ with values in $M_{3}^{+}(\mathbb{R})$ is thus constructed, for all $\mathbf{x}$ in $\Omega^{\text {meso }}$, as

$$
\left[\mathbf{C}^{2 D}(\mathbf{x})\right]=\left[\mathbf{S}^{2 D}(\mathbf{x})\right]^{-1} .
$$

Consequently, the hyperparameter $\mathbf{b}$ of the prior stochastic model of the apparent elasticity random field $\left[\mathbf{C}^{2 D}(\mathbf{b})\right]=\left\{\left[\mathbf{C}^{2 D}(\mathbf{x} ; \mathbf{b})\right], \mathbf{x} \in \Omega^{\text {meso }}\right\}$ are $\mathbf{b}=(\delta, \ell$, algebraically independent parameters spanning the symmetric matrix $\left.\left[\underline{S}^{2 D}\right]\right)$.

\subsubsection{Multi-objective optimization problem for $2 D$ plane stresses}

The identification of hyperparameter $\mathbf{b}$ is carried out in searching for the optimal values $\mathbf{a}^{\text {macro }}$ and $\mathbf{b}^{\text {meso }}$ which solve the following multi-objective minimization problem

$$
\left(\mathbf{a}^{\text {macro }}, \mathbf{b}^{\text {meso }}\right)=\underset{\mathbf{a} \in \mathcal{A}^{\text {macro }}, \mathbf{b} \in \mathcal{B}^{\text {meso }}}{\arg \min } \mathcal{I}(\mathbf{a}, \mathbf{b}),
$$

where $\mathcal{A}^{\text {macro }}$ and $\mathcal{B}^{\text {meso }}$ are the sets of the admissible values for $\mathbf{a}$ and $\mathbf{b}$, and where the components $\mathcal{I}_{1}(\mathbf{a}), \mathcal{I}_{2}(\mathbf{b})$ and $\mathcal{I}_{3}(\mathbf{a}, \mathbf{b})$ of vector $\mathcal{I}(\mathbf{a}, \mathbf{b})$ are defined by Eqs. (5), (7) and (11). Concerning the multi-objective optimization, if the material at macroscale is assumed to be transverse isotropic and in 2D plane stresses, then the vector-valued parameter $\mathbf{a}=\left(E_{T}^{\text {macro }}, \nu_{T}^{\text {macro }}\right)$ corresponds to the transverse Young modulus and the transverse Poisson coefficient at macroscale. The hyperparameter $\mathbf{b}$ will be defined later. The stochastic boundary value problem at mesoscale is solved in using the finite element method. The Monte Carlo numerical method is used to calculate the indicators $\mathcal{I}_{2}(\mathbf{b})$ and $\mathcal{I}_{3}(\mathbf{a}, \mathbf{b})$ with a number $n_{s}$ of statistical independent realizations of the random elasticity field $\left\{\left[\mathbf{C}^{2 D}(\mathbf{x})\right], \mathbf{x} \in \Omega^{\text {meso }}\right\}$. The multi-objective optimization problem defined by Eq. (13) is solved by using a genetic algorithm and the Pareto front is iteratively constructed at each generation of the genetic algorithm. The initial value of parameter a has been set to $\mathbf{a}^{(0)}$ and corresponds to the solution of the following partial optimization problem: 
$\mathbf{a}^{(0)}=\arg \min \mathcal{I}_{1}(\mathbf{a})$ for $\mathbf{a} \in \mathcal{A}^{\text {macro, }}$, which is solved with the simplex algorithm. The optimal value $\mathbf{b}^{\text {meso }}$ is then chosen as the point on the Pareto front that minimizes the distance between the Pareto front and the origin.

\section{Validation of the method for $2 \mathrm{D}$ plane stresses}

The validation is performed within the framework of the linear elasticity for $2 \mathrm{D}$ plane stresses. It should be noted that the two directions are observed when the displacement fields are measured at macroscale and at mesoscale with a camera.

\subsection{Construction of a simulated "experimental" database}

In order to validate the methodology, some "experimental" measurements are simulated for the macroscale and the mesoscale by using a computational model. The $2 \mathrm{D}$ domain, $\Omega^{\text {macro }}$, is defined in the plane $\left(O x_{1} x_{2}\right)$ as a square whose dimension of the edge is $h=10^{-2} \mathrm{~m}$. At mesoscale, the material is heterogeneous, anisotropic and linear elastic. A line force directed along $-x_{2}$, with an intensity of $5 \times 10^{-2} \mathrm{~N} / \mathrm{m}$, is applied on the edge $x_{2}=h$. The edge $x_{2}=0$ is fixed. A 2D plane stress state is assumed. At mesoscale, the $2 \mathrm{D}$ apparent elasticity field is constructed as a realization of the prior stochastic model of the random field $\left[\mathbf{C}^{2 D}(\mathbf{b})\right]$ with $\ell=1.25 \times 10^{-4} \mathrm{~m}, \delta=0.4$ and where the entries of $\left[\underline{S}^{2 D}\right]$ are defined using $\left[\underline{S}^{\text {meso }}\right]$ which corresponds to a transverse isotropic linear elastic medium,

$$
\left[\underline{S}^{\text {meso }}\right]=\left[\begin{array}{cccccc}
\frac{1}{E_{T}} & -\frac{\nu_{T}}{E_{T}} & 0 & -\frac{\nu_{L}}{E_{L}} & 0 & 0 \\
-\frac{\nu_{T}}{E_{T}} & \frac{1}{E_{T}} & 0 & 0 & 0 & 0 \\
0 & 0 & \frac{2\left(1+\nu_{T}\right)}{E_{T}} & 0 & 0 & 0 \\
-\frac{\nu_{L}}{E_{L}} & -\frac{\nu_{L}}{E_{L}} & 0 & \frac{1}{E_{L}} & 0 & 0 \\
0 & 0 & 0 & 0 & \frac{1}{G_{L}} & 0 \\
0 & 0 & 0 & 0 & 0 & \frac{1}{G_{L}}
\end{array}\right]
$$

with $E_{L}=15.8 \times 10^{9} \mathrm{~Pa}, E_{T}=9.9 \times 10^{9} \mathrm{~Pa}, G_{L}=5.2 \times 10^{9} \mathrm{~Pa}, \nu_{L}=0.31$ and $\nu_{T}=0.38$. Consequently, we have

$$
\left[\underline{S}^{2 D}\right]=\left[\begin{array}{ccc}
\frac{1}{E_{T}} & -\frac{\nu_{T}}{E_{T}} & 0 \\
-\frac{\nu_{T}}{E_{T}} & \frac{1}{E_{T}} & 0 \\
0 & 0 & \frac{2\left(1+\nu_{T}\right)}{E_{T}}
\end{array}\right] .
$$

Thus, the vector-valued hyperparameter $\mathbf{b}$ is written as $\mathbf{b}=\left(\delta, \ell, E_{T}, \nu_{T}\right)$. At mesoscale, the realization of the apparent elasticity random field is simulated on 
the whole domain $\Omega^{\text {macro }}$.

A computational model is constructed with the finite element method and a regular finite element mesh with one million quadrangle elements (1,000 along $x_{1}$ and 1, 000 along $x_{2}$, see Fig. 1, left). The strain field is numerically simulated in using a finite element interpolation in a regular grid of nodes with a mesoscale resolution on the whole domain $\Omega^{\text {macro }}$ (see Fig. 1, center). Measurements of the strain field $\varepsilon_{\exp }^{\text {macro }}$ is simulated at macroscale in extracting the values of the displacement field in a regular grid of $10 \times 10$ nodes and in using a finite element interpolation (see Fig. 1, upper right). In addition, in the subdomain defined as a square with dimension $10^{-3} \mathrm{~m}$ (mesoscale), the measurements of the strain field $\varepsilon_{\exp }^{\text {meso }}$ are simulated at mesoscale in extracting the values of the displacement field in a regular grid of $100 \times 100$ nodes and in using a finite element interpolation (see Fig. 1, lower right).

Figure 2 shows the values of $\left\{\varepsilon_{\exp }^{\text {macro }}\right\}_{22}$ for the simulated experimental strain field at macroscale with a resolution $10 \times 10$. The square in black dashed line represents the considered mesoscale subdomain. Figure 3 shows the values of $\left\{\varepsilon_{\exp }^{\text {meso }}\right\}_{22}$ for the simulated experimental strain field at mesoscale with a resolution $100 \times 100$.

\subsection{Numerical results and validation}

At macroscale, the prior model of the material is chosen as a transverse isotropic model. Consequently, for $2 \mathrm{D}$ plane stresses, the vector-valued parameter $\mathbf{a}=$ $\left(E_{T}^{\text {macro }}, \nu_{T}^{\text {macro }}\right)$ is made up of the transverse Young modulus and the transverse Poisson coefficient. The optimal value of $\mathbf{a}=\left(E_{T}^{\text {macro }}, \nu_{T}^{\text {macro }}\right)$ is $\mathbf{a}^{\text {macro }}=(9.565 \times$ $\left.10^{9} \mathrm{~Pa}, 0.3987\right)$. The finite element mesh of the subdomain at mesoscale is (i) cartesian with a constant size in directions $x_{1}$ and $x_{2}$, and (ii) made up of $9,801=99 \times 99$ finite elements with 4 nodes. The total number of Gauss points associated with the finite element mesh is 39,204 . The convergence of the statistical estimators is reached for 500 independent realizations of the random elasticity field $\left[\mathbf{C}^{2 D}\right]$. An initial population size of 50 is used for the solving the optimization problem defined by Eq. (13) with the genetic algorithm. Less than 100 generations has been enough for constructing the Pareto front which is iteratively constructed, at each generation of the genetic algorithm. The value of $\mathbf{a}^{\text {macro }}$ is almost unchanged through the iterations when the multi-objective problem is solved. The computation has been performed with 50 cores with a $\mathrm{CPU}$ at $2 \mathrm{GHz}$, and has required 120 hours of CPU time. 


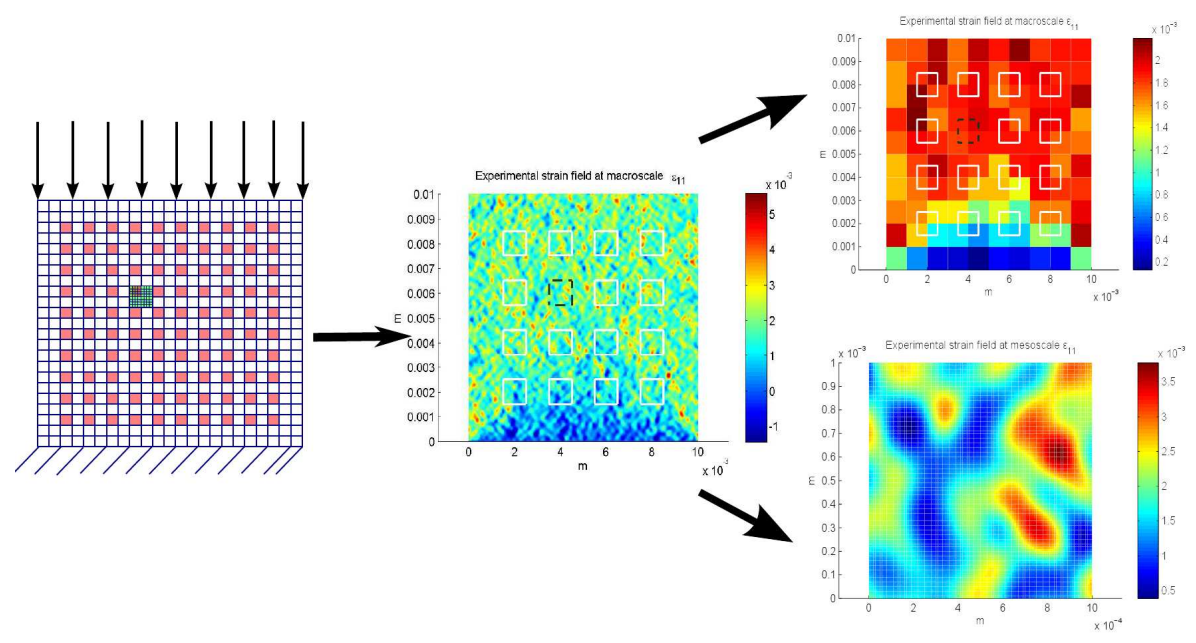

Figure 1: Description of the methodology for the construction of the simulated experimental measurements in using the finite element method at macroscale and at mesoscale: FE model of the specimen at macroscale with a mesoscale resolution (left); component $\{11\}$ of the strain field at macroscale with a mesoscale resolution (center); component $\{11\}$ of the strain field at macroscale with a macroscale resolution (upper right); component $\{11\}$ of the strain field at mesoscale with a mesoscale resolution (lower right).

Table 1 shows the values of $\mathbf{b}=\left(\ell, \delta, E_{T}, \nu_{T}\right)$ for each point of the Pareto front displayed in Fig. 4. The optimal values correspond to the points 5, 6, 7, 8 and 9 where points 6 et 7 are close. The optimal value $\mathbf{b}^{\text {meso }}$ is such that $\ell^{\text {meso }}=$ $9.66 \times 10^{-5} \mathrm{~m}, \delta^{\text {meso }}=0.37, E_{T}^{\text {meso }}=1.023 \times 10^{10} \mathrm{~Pa}, \nu_{T}^{\text {meso }}=0.376$. This result yields a validation of the proposed methodology since this identified optimal value $\mathbf{b}^{\text {meso }}$ is very close to the value $\mathbf{b}$ that has been used to construct the simulated experimental database for which $\ell=1.25 \times 10^{-4} \mathrm{~m}, \delta=0.4, E_{T}=9.9 \times 10^{9} \mathrm{~Pa}$, $\nu_{T}=0.38$. 


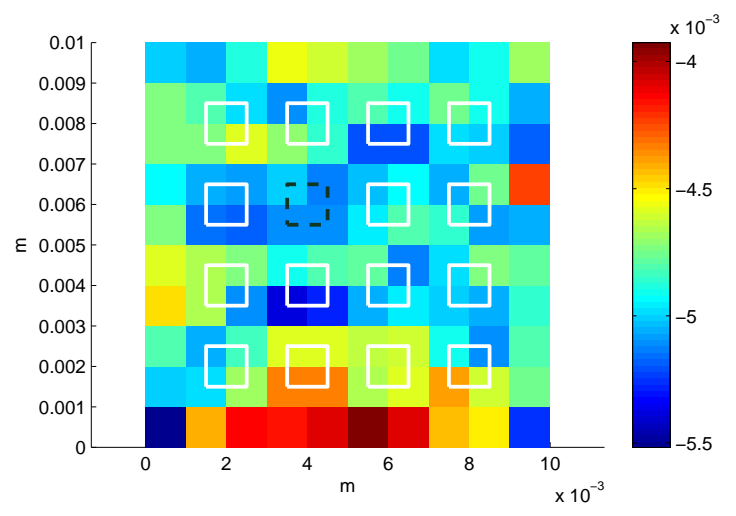

Figure 2: Component $\left\{\varepsilon_{\exp }^{\text {macro }}\right\}_{22}$ of the simulated experimental strain field at macroscale with a resolution $10 \times 10$.

\section{Application of the method with multiscale experimental measurements}

In this section, results are presented for the method with real multiscale experimental measurements obtained by using a CCD camera coupled with a microscope and the digital image correlation (DIC) method for measuring the displacement fields on one sample of cortical bone at macroscale and at mesoscale. The measurements have been carried out at the Laboratory of Solid Mechanics of Ecole Polytechnique, and all the details concerning these multiscale experiments are presented in [42].

\subsection{Multiscale experimental database}

The DIC method allows the displacement field on an enlightened face of a sample under external loads to be measured for a given resolution of the camera and of the speckle pattern. The displacement field is obtained by comparing two images of the enlightened face, the first one when the sample is undeformed (image of reference) and the second image when the sample is deformed by the external loads (deformed image). Fig. 5 shows the images obtained for a sample of cortical bone. The comparison of the images is based on the correlation of the images under the assumption that the contrast is conserved locally in a vicinity of the points where the displacement is measured. 


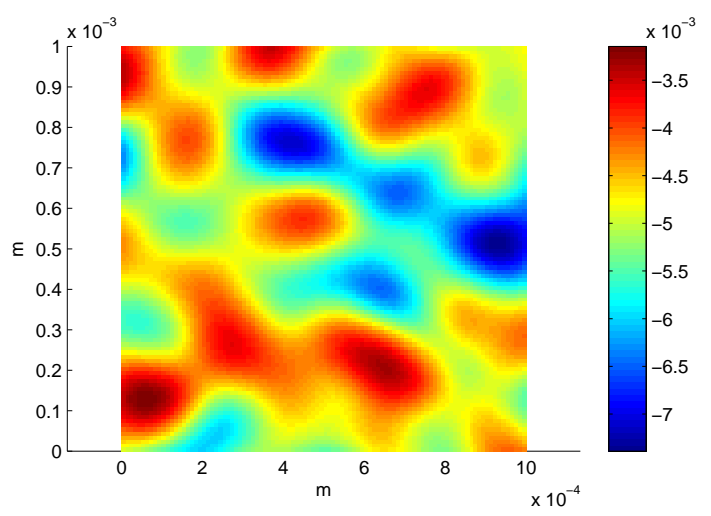

Figure 3: Component $\left\{\varepsilon_{\exp }^{\text {meso }}\right\}_{22}$ of the simulated experimental strain field at mesoscale with a resolution $100 \times 100$.

The experimental configuration for one sample of cortical bone, which came from beef femur, is the same as for the example presented in section 4. The uniaxial load had to be limited to $9000 \mathrm{~N}$ in order to be in the elastic domain of the sample. A resolution of $10 \times 10$ points at macroscale, and a resolution of $100 \times 100$ points at mesoscale are used to identify the displacement fields at mesoscale and at macroscale. Fig. 6 and Fig. 7 display the identified displacement fields along directions $x_{1}$ and $x_{2}$ at macroscale. Fig. 8 and Fig. 9 display the identified displacement fields along directions $x_{1}$ and $x_{2}$ at mesoscale.

\subsection{Numerical results}

The optimal value of $\mathbf{a}=\left(E_{T}^{\text {macro }}, \nu_{T}^{\text {macro }}\right)$ is $\mathbf{a}^{\text {macro }}=\left(6.74 \times 10^{9} \mathrm{~Pa}, 0.32\right)$. The parameters of the method (finite element mesh size, number of independent realizations, initial population size for the genetic algorithm) are the same than in Section 4.2. Less than 100 generations has been enough for constructing the Pareto front which is iteratively constructed, at each generation of the genetic algorithm.

For each point of the Pareto front, displayed in Fig. 10, the values of $\mathbf{b}=\left(\ell, \delta, E_{T}, \nu_{T}\right)$ are summarized in Tab 2. The ninth point of the Pareto front minimizes the distance to the origin. Its optimal components are $\ell^{\text {meso }}=5.06 \times 10^{-5} \mathrm{~m}, \delta^{\text {meso }}=$ 
Table 1: Optimization results of the genetic algorithm

\begin{tabular}{ccccccc}
\hline $\mathrm{k}$ & $\mathcal{I}_{2}(\mathbf{b})$ & $\mathcal{I}_{3}(\mathbf{a}, \mathbf{b})$ & $\ell$ & $\delta$ & $E_{T}$ & $\nu_{T}$ \\
1 & $5.006529 \times 10^{-9}$ & $2.311672 \times 10^{-1}$ & $1.886667 \times 10^{-4}$ & 0.400000 & $1.023000 \times 10^{10}$ & 0.392667 \\
2 & $5.006529 \times 10^{-9}$ & $9.477024 \times 10^{-2}$ & $2.500000 \times 10^{-4}$ & 0.400000 & $1.023000 \times 10^{10}$ & 0.392667 \\
3 & $5.010827 \times 10^{-9}$ & $9.469903 \times 10^{-2}$ & $9.666667 \times 10^{-5}$ & 0.366667 & $1.023000 \times 10^{10}$ & 0.376200 \\
4 & $5.132208 \times 10^{-9}$ & $9.201960 \times 10^{-2}$ & $1.273333 \times 10^{-4}$ & 0.383333 & $1.023000 \times 10^{10}$ & 0.392667 \\
5 & $5.240100 \times 10^{-9}$ & $3.467300 \times 10^{-2}$ & $9.666667 \times 10^{-5}$ & 0.366667 & $1.023000 \times 10^{10}$ & 0.359733 \\
6 & $5.259407 \times 10^{-9}$ & $2.455275 \times 10^{-2}$ & $5.066667 \times 10^{-5}$ & 0.350000 & $8.943000 \times 10^{9}$ & 0.293867 \\
7 & $5.259407 \times 10^{-9}$ & $2.455275 \times 10^{-2}$ & $9.666667 \times 10^{-5}$ & 0.366667 & $1.023000 \times 10^{10}$ & 0.376200 \\
8 & $5.386876 \times 10^{-9}$ & $2.064010 \times 10^{-2}$ & $5.066667 \times 10^{-5}$ & 0.350000 & $8.943000 \times 10^{9}$ & 0.310333 \\
9 & $5.490529 \times 10^{-9}$ & $1.968774 \times 10^{-2}$ & $5.066667 \times 10^{-5}$ & 0.350000 & $1.237500 \times 10^{10}$ & 0.293867 \\
10 & $6.57386 \times 10^{-9}$ & $1.962839 \times 10^{-2}$ & $2.193333 \times 10^{-4}$ & 0.400000 & $1.023000 \times 10^{10}$ & 0.392667 \\
11 & $6.895467 \times 10^{-9}$ & $1.885624 \times 10^{-2}$ & $2.500000 \times 10^{-4}$ & 0.383333 & $1.023000 \times 10^{10}$ & 0.392667 \\
12 & $7.254986 \times 10^{-9}$ & $1.759584 \times 10^{-2}$ & $2.500000 \times 10^{-4}$ & 0.333333 & $1.023000 \times 10^{10}$ & 0.392667 \\
13 & $7.567184 \times 10^{-9}$ & $1.688894 \times 10^{-2}$ & $9.666667 \times 10^{-5}$ & 0.383333 & $1.023000 \times 10^{10}$ & 0.392667 \\
14 & $7.996816 \times 10^{-9}$ & $1.623193 \times 10^{-2}$ & $2.000000 \times 10^{-5}$ & 0.350000 & $8.943000 \times 10^{9}$ & 0.310333 \\
15 & $9.129340 \times 10^{-9}$ & $1.507042 \times 10^{-2}$ & $2.500000 \times 10^{-4}$ & 0.366667 & $1.023000 \times 10^{10}$ & 0.392667 \\
16 & $9.368447 \times 10^{-9}$ & $1.333442 \times 10^{-2}$ & $1.273333 \times 10^{-4}$ & 0.266667 & $1.023000 \times 10^{10}$ & 0.392667 \\
\hline
\end{tabular}

$0.28, E_{T}^{\text {meso }}=6.96 \times 10^{9} \mathrm{~Pa}, \nu_{T}^{\text {meso }}=0.37$. This optimal solution yields a spatial correlation length equal to $5.06 \times 10^{-5} \mathrm{~m}$ which is in agreement with the assumption introduced concerning the separation of the scales. This length is also of the same order of magnitude than the distance between adjacent lamellae or osteons in cortical bovine femur.

Table 2: Optimization in using the genetic algorithm with experimental data

\begin{tabular}{ccccccc}
\hline $\mathrm{k}$ & $\mathcal{I}_{2}(\mathbf{b})$ & $\mathcal{I}_{3}(\mathbf{a}, \mathbf{b})$ & $\ell$ & $\delta$ & $E_{T}$ & $\nu_{T}$ \\
1 & $9.905566 \times 10^{-4}$ & $1.130759 \times 10^{-1}$ & $5.066667 \times 10^{-5}$ & 0.300000 & $6.964455 \times 10^{9}$ & 0.399180 \\
2 & $9.905566 \times 10^{-4}$ & $5.529077 \times 10^{-2}$ & $5.066667 \times 10^{-5}$ & 0.300000 & $6.964455 \times 10^{9}$ & 0.385342 \\
3 & $9.905566 \times 10^{-4}$ & $5.529077 \times 10^{-2}$ & $5.066667 \times 10^{-5}$ & 0.300000 & $6.964455 \times 10^{9}$ & 0.399180 \\
4 & $9.923024 \times 10^{-4}$ & $4.566584 \times 10^{-2}$ & $5.066667 \times 10^{-5}$ & 0.300000 & $6.964455 \times 10^{9}$ & 0.371504 \\
5 & $9.942891 \times 10^{-4}$ & $3.721737 \times 10^{-2}$ & $5.066667 \times 10^{-5}$ & 0.300000 & $6.964455 \times 10^{9}$ & 0.371504 \\
6 & $9.942891 \times 10^{-4}$ & $3.721737 \times 10^{-2}$ & $5.066667 \times 10^{-5}$ & 0.266667 & $6.964455 \times 10^{9}$ & 0.371504 \\
7 & $9.943808 \times 10^{-4}$ & $3.707802 \times 10^{-2}$ & $5.066667 \times 10^{-5}$ & 0.300000 & $6.380340 \times 10^{9}$ & 0.399180 \\
8 & $9.947595 \times 10^{-4}$ & $3.698333 \times 10^{-2}$ & $1.426667 \times 10^{-4}$ & 0.383333 & $6.672398 \times 10^{9}$ & 0.316151 \\
9 & $9.965090 \times 10^{-4}$ & $3.092503 \times 10^{-2}$ & $5.066667 \times 10^{-5}$ & 0.283333 & $6.964455 \times 10^{9}$ & 0.371504 \\
10 & $9.989547 \times 10^{-4}$ & $2.826733 \times 10^{-2}$ & $2.040000 \times 10^{-4}$ & 0.416667 & $6.672398 \times 10^{9}$ & 0.343827 \\
11 & $1.009211 \times 10^{-3}$ & $2.125592 \times 10^{-2}$ & $2.040000 \times 10^{-4}$ & 0.483333 & $6.672398 \times 10^{9}$ & 0.343827 \\
12 & $1.011463 \times 10^{-3}$ & $2.064572 \times 10^{-2}$ & $5.066667 \times 10^{-5}$ & 0.300000 & $6.964455 \times 10^{9}$ & 0.357666 \\
13 & $1.019979 \times 10^{-3}$ & $1.925469 \times 10^{-2}$ & $5.066667 \times 10^{-5}$ & 0.300000 & $6.964455 \times 10^{9}$ & 0.343827 \\
14 & $1.023436 \times 10^{-3}$ & $1.901348 \times 10^{-2}$ & $2.040000 \times 10^{-4}$ & 0.500000 & $6.672398 \times 10^{9}$ & 0.343827 \\
15 & $1.024812 \times 10^{-3}$ & $2.645432 \times 10^{-3}$ & $2.040000 \times 10^{-4}$ & 0.433333 & $6.672398 \times 10^{9}$ & 0.343827 \\
\hline
\end{tabular}

\section{Conclusions}

In the framework of the linear elasticity, a multiscale inverse statistical method has been presented for the identification of a stochastic model of the apparent elasticity random field at mesoscale for a heterogeneous microstructure using experimental 


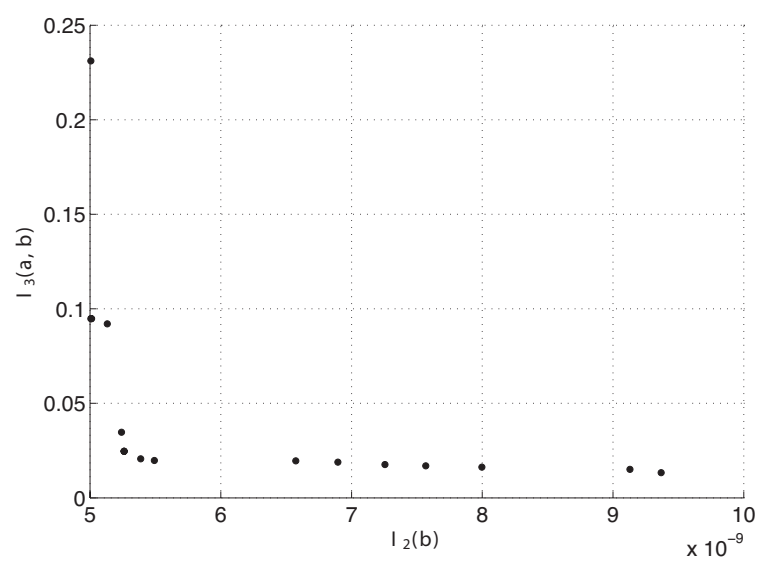

Figure 4: Pareto front for the numerical indicators $\mathcal{I}_{2}(\mathbf{b})$ and $\mathcal{I}_{3}(\mathbf{a}, \mathbf{b})$.
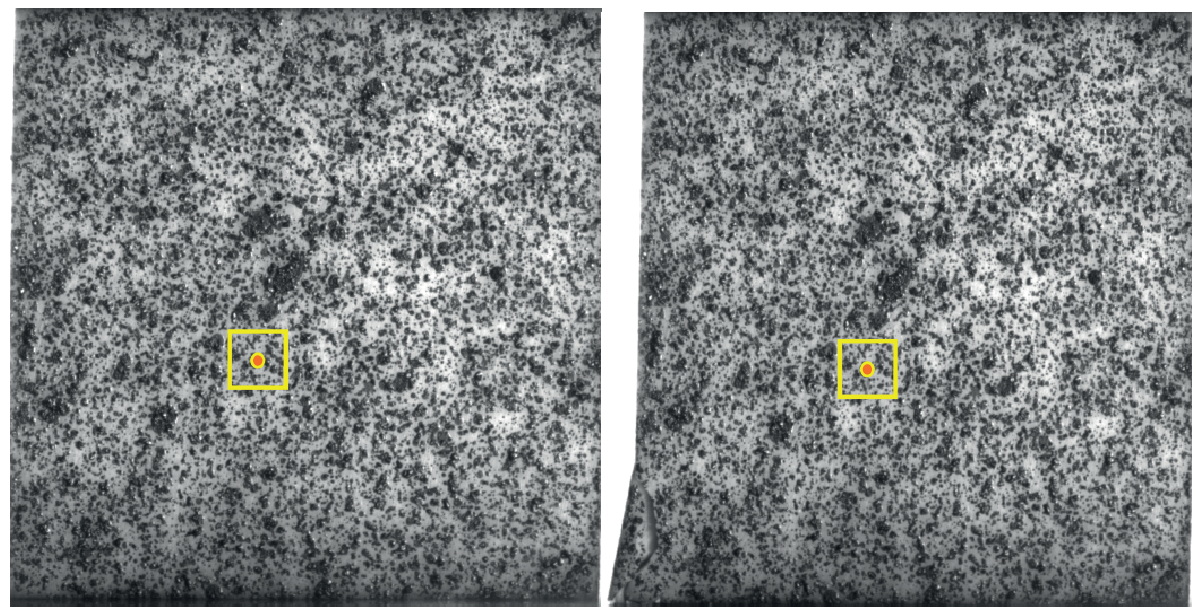

Figure 5: Comparison between a reference image (left) and a deformed image (right) at macroscale for a cubic cortical bovine bone sample.

measurements at macroscale and at mesoscale. A prior stochastic model depending of a hyperparameter has been proposed for the apparent elasticity random field at mesoscale in the case of 2D plane stresses. The identification procedure has been formulated as a multi-objective minimization problem with respect to the parameter of the elastic model at macroscale and the hyperparameter of the prior stochastic model at mesoscale. The optimal value of the parameter and the hyperparameter corresponds to the point that minimizes the distance of a Pareto 

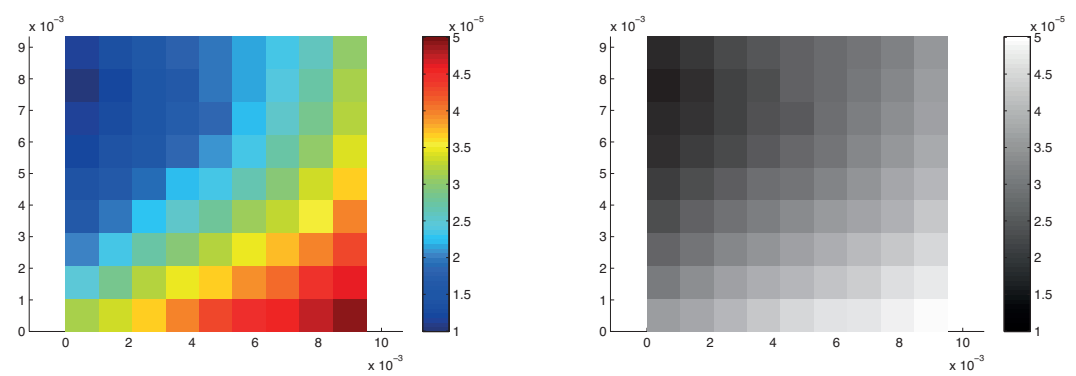

Figure 6: Component $\left\{\mathbf{u}_{\exp }^{\text {macro }}\right\}_{1}$ in direction $x_{1}$ (horizontal) for the experimental displacement at macroscale: in color (left) and in black and white (right).
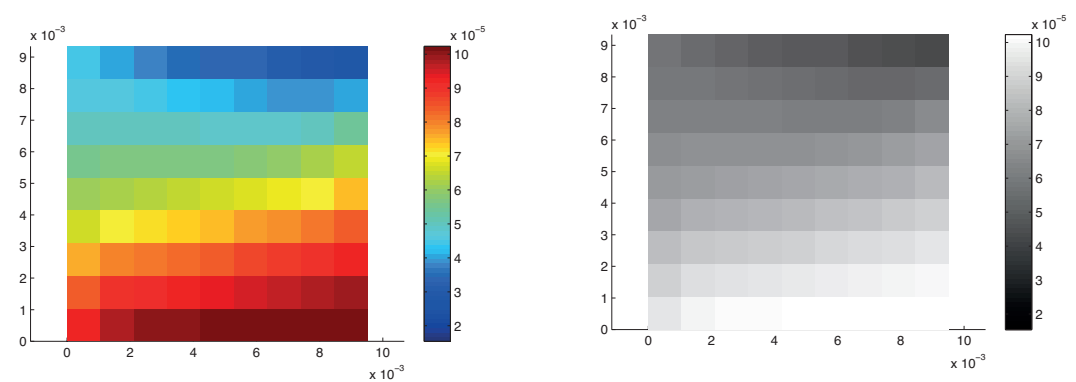

Figure 7: Component $\left\{\mathbf{u}_{\exp }^{\text {macro }}\right\}_{2}$ in direction $x_{2}$ (vertical) for the experimental displacement at macroscale: in color (left) and in black and white (right).
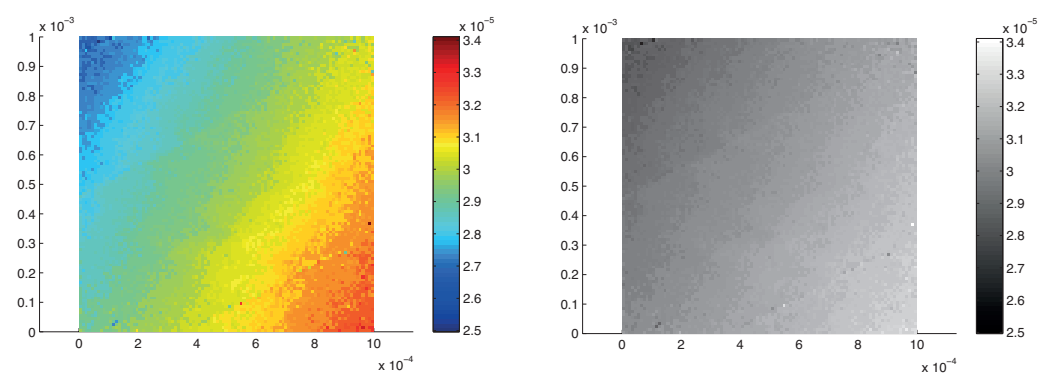

Figure 8: Component $\left\{\mathbf{u}_{\mathrm{exp}}^{\text {meso }}\right\}_{1}$ in direction $x_{1}$ (horizontal) for the experimental displacement at mesoscale: in color (left) and in black and white (right).

front to the origin. The proposed statistical inverse method has been validated with a simulated experimental database and results have been presented in the 

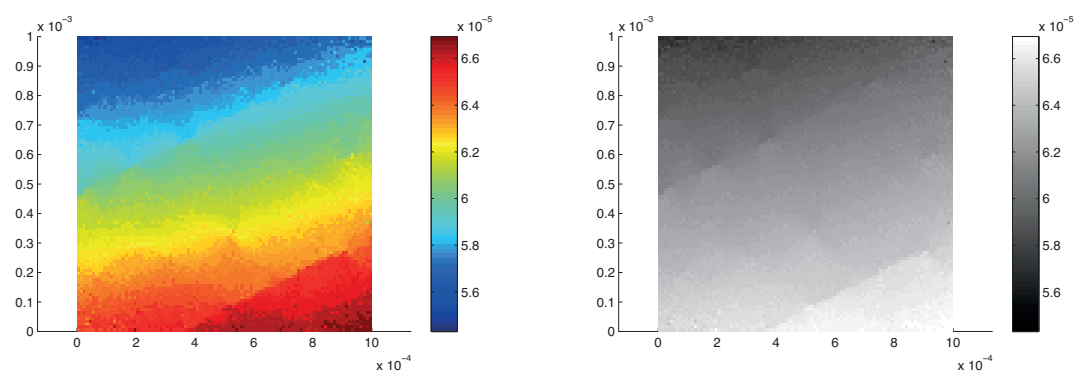

Figure 9: Component $\left\{\mathbf{u}_{\exp }^{\text {meso }}\right\}_{2}$ in direction $x_{2}$ (vertical) for the experimental displacement at mesoscale: in color (left) and in black and white (right).

case of experimental measurements obtained by the DIC method on one sample of cortical bone observed by a CCD camera at both macroscale and mesoscale.

\section{Acknowledgement}

This research was supported by the "Agence Nationale de la Recherche", Contract TYCHE, ANR-2010-BLAN-0904.

\section{References}

[1] Arnst, M., Clouteau, D., and Bonnet, M., Inversion of probabilistic structural models using measured transfer functions, Computer Methods in Applied Mechanics and Engineering, vol. 197, pp. 589-608, 2008.

[2] Avril, S., Bonnet, M., Bretelle, A.-S., Grédiac, M., Hild, F., Ienny, P., Latourte, F., Lemosse, D., Pagano, S., Pagnacco, E., and Pierron, F., Overview of identification methods of mechanical parameters based on full-field measurements, Experimental Mechanics, vol. 48, pp. 381-402, 2008.

[3] Avril, S. and Pierron, F., General framework for the identification of constitutive parameters from full-field measurements in linear elasticity, International Journal of Solids and Structures, vol. 44, pp. 4978-5002, 2007.

[4] Avril, S., Pierron, F., Pannier, Y., and Rotinat, R., Stress reconstruction and constitutive parameter identification in plane-stress elastoplastic problems using surface measurements of deformation fields, pp. 403-419, 2008. 


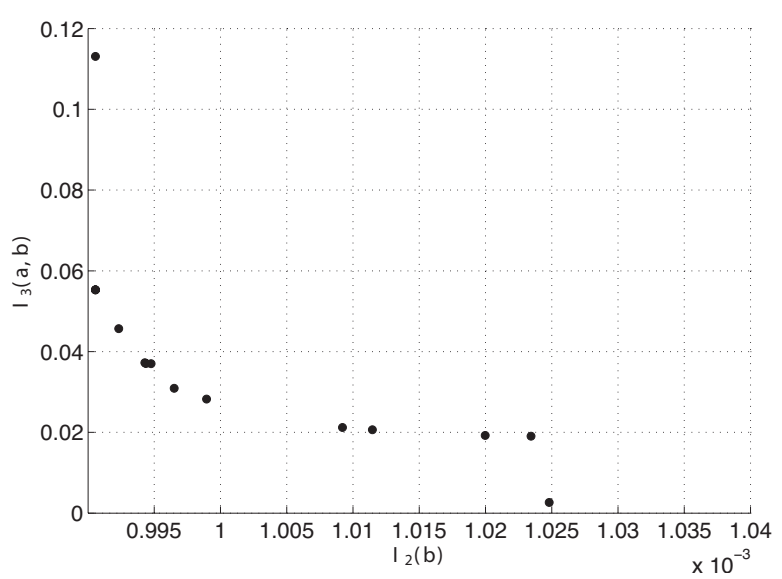

Figure 10: Pareto front for the two numerical indicators $\mathcal{I}_{2}(\mathbf{b})$ and $\mathcal{I}_{3}(\mathbf{a}, \mathbf{b})$ for the experimental identification.

[5] Babuska, I., Nobile, F., and Tempone, R., A stochastic collocation method for elliptic partial differential equations with random input data, SIAM J. Numer. Anal., vol. 45 (3), pp. 1005-1034, 2007.

[6] Babuska, I., Tempone, R., and Zouraris, G. E., Solving elliptic boundary value problems with uncertain coefficients by the finite element method: the stochastic formulation, Comput. Methods Appl. Mech. Engrg., vol. 194 (12-16), pp. 1251-1294, 2005.

[7] Baxter, S. C. and Graham, L. L., Characterization of random composites using a moving window technique, ASCE J Engng Mech, vol. 126(4), pp. 389-404, 2000 .

[8] Besnard, G., Hild, F., and Roux, S., Finite-element displacement fields analysis from digital images: Application to portevin-le chatelier bands, Experimental Mechanics, vol. 46, pp. 789-803, 2006.

[9] Bonnet, M. and Constantinescu, A., Inverse problems in elasticity, Inverse Probl, vol. 21, pp. R1-R50, 2005.

[10] Bornert, M., Brémand, F., Doumalin, P., Dupré, J.-C., Fazzini, M., Grédiac, M., Hild, F., Mistou, S., Molimard, J., Orteu, J.-J., Robert, L., Surrel, Y., 
Vacher, P., and Wattrisse, B., Assessment of digital image correlation measurement errors: Methodology and results, Experimental Mechanics, vol. 49, pp. 353-370, 2009.

[11] Bornert, M., Vales, F., Gharbi, H., and Nguyen Minh, D., Multiscale fullfield strain measurements for micromechanical investigations of the hydromechanical behaviour of clayey rocks, Strain, vol. 46, pp. 33-46, 2010.

[12] Calloch, S., Dureisseix, D., and Hild, F., Identification de modèles de comportement de matériaux solides: utilisation d'essais et de calculs, Technol Form, vol. 100, pp. 36-41, 2002.

[13] Chevalier, L., Calloch, S., Hild, F., and Marco, Y., Digital image correlation used to analyze the multiaxial behavior of rubber-like materials, European Journal of Mechanics A-Solids, vol. 20, pp. 169-187, 2001.

[14] Clouteau, D., Cottereau, R., and Lombaert, G., Dynamics of structures coupled with elastic media - a review of numerical models and methods, Journal of Sound and Vibration, vol. 332, pp. 2415-2436, 2013.

[15] Collins, J. C., Hart, G. C., and Kennedy, B., Statistical identification of structures, AIAA J, vol. 12, pp. 185-190, 1974.

[16] Constantinescu, A., On the identification of elastic moduli from displacement-force boundary measurements, Inverse Probl Eng, vol. 1, pp. 293-315, 1995.

[17] Das, S., Ghanem, R., and Finette, S., Polynomial chaos representation of spatiotemporal random field from experimental measurements, J. Comput. Phys., vol. 228, pp. 8726-8751, 2009.

[18] Das, S., Ghanem, R., and Spall, J., Asymptotic sampling distribution for polynomial chaos representation of data: a maximum-entropy and fisher information approach, SIAM J. Sci. Comput., vol. 30 (5), pp. 2207-2234, 2008.

[19] Desceliers, C., Ghanem, R., and Soize, C., Maximum likelihood estimation of stochastic chaos representations from experimental data, International Journal For Numerical Methods In Engineering, vol. 66, pp. 978-1001, 2006.

[20] Desceliers, C., Soize, C., and Ghanem, R., Identification of chaos representations of elastic properties of random media using experimental vibration tests, Comput.Mech., vol. 39(6), pp. 831-838, 2007. 
[21] Desceliers, C., Soize, C., Naili, S., and Haiat, G., Probabilistic model of the human cortical bone with mechanical alterations in ultrasonic range, Mechanical Systems and Signal Processing, vol. 32, pp. 170-177, 2012.

[22] Geymonat, G., Hild, F., and Pagano, S., Identification of elastic parameters by displacement field measurement, Comptes Rendus Mécanique, vol. 330, pp. 403-408, 2002.

[23] Geymonat, G. and Pagano, S., Identification of mechanical properties by displacement field measurement: a variational approach, Meccanica, vol. 38, pp. 535-545, 2003.

[24] Graham, L. L., Gurley, K., and Masters, F., Non-gaussian simulation of local material properties based on a moving-window technique, Prob. Eng. Mechanics, vol. 18, pp. 223-234, 2003.

[25] Guilleminot, J. and Soize, C., Non-gaussian positive-definite matrix-valued random fields with constrained eigenvalues: Application to random elasticity tensors with uncertain material symmetries, International Journal for Numerical Methods in Engineering, vol. 88, pp. 1128-1151, 2011.

[26] Guilleminot, J. and Soize, C., Generalized stochastic approach for constitutive equation in linear elasticity: a random matrix model, International Journal for Numerical Methods in Engineering, vol. 90, pp. 613-635, 2012.

[27] Guilleminot, J. and Soize, C., Probabilistic modeling of apparent tensors in elastostatics: A maxent approach under material symmetry and stochastic boundedness constraints, Probabilistic Engineering Mechanics, vol. 28, pp. $118-124,2012$.

[28] Guilleminot, J. and Soize, C., Stochastic model and generator for random fields with symmetry properties: application to the mesoscopic modeling of elastic random media, Multiscale Modeling and Simulation (A SIAM Interdisciplinary Journal), vol. 11, pp. 840-870, 2013.

[29] Guilleminot, J., Soize, C., and Kondo, D., Mesoscale probabilistic models for the elasticity tensor of fiber reinforced composites: Experimental identification and numerical aspects, Mechanics of Materials, vol. 41, pp. 1309-1322, 2009. 
[30] Hild, F., CORRELI ${ }^{L M T}$ : a software for displacement field measurements by digital image correlation, , 2002.

[31] Hild, F., Périé, J.-N., and Coret, M., Mesure de champs de déplacements 2D par intercorrélation d'images: CORRELI 2D, , 1999.

[32] Hild, F., Raka, B., Baudequin, M., Roux, S., and Cantelaube, F., Multiscale displacement field measurements of compressed mineral-wool samples by digital image correlation, Applied Optics, vol. 41, pp. 6815-6828, 2002.

[33] Hild, F. and Roux, S., Digital image correlation: from displacement measurement to identification of elastic properties - a review, Strain, vol. 42, pp. 69-80, 2006.

[34] Hild, F. and Roux, S., Comparison of local and global approaches to digital image correlation, Experimental Mechanics, vol. 52, pp. 1503-1519, 2012.

[35] Jeulin, D., Microstructure modeling by random textures, Journal de Microscopie et de Spectroscopie Electroniques, vol. 12, pp. 133140, 1987.

[36] Jeulin, D., Morphological modeling of images by sequential random functions, Signal Processing, vol. 16, pp. 403431, 1989.

[37] Jeulin, D., Caractérisation morphologique et modèles de structures aléatoires. In Homogénéisation en mécanique des matériaux 1, Hermès Science Publications, 2001.

[38] Kahnjetter, Z. L., Jha, N. K., and Bhatia, H., Optimal image correlation in experimental mechanics, Optical Engineering, vol. 33, pp. 1099-1105, 1994.

[39] Kaipio, J. and Somersalo, E., Statistical and Computational Inverse Problems, Springer-Verlag, New York, 2005.

[40] Lawson, C. L. and Hanson, R. J., Solving Least Squares Problems, PrenticeHall, 1974.

[41] Madi, K., Forest, S., Boussuge, M., Gailliegue, S., Lataste, E., Buffiere, J.Y., Bernard, D., and Jeulin, D., Finite element simulations of the deformation of fused-cast refractories based on x-ray computed tomography, Computational Materials Science, vol. 39, pp. 224-229, 2007. 
[42] Nguyen, M. T., Allain, J. M., Gharbi, H., Desceliers, C., and Soize, C., Experimental measurements for identification of the elasticity field at mesoscale of a heterogeneous microstructure by multiscale digital image correlation, $E x$ perimental Mechanics, Submitted September 2014.

[43] Nouy, A., and Soize, C., Random fields representations for stochastic elliptic boundary value problems and statistical inverse problems, European Journal of Applied Mathematics, online, pp. 1-35, 21 March 2014.

[44] Perrin, G., Soize, C., Duhamel, D., and Funfschilling, C., Identification of polynomial chaos representations in high dimension from a set of realizations, SIAM Journal on Scientific Computing, vol. 34, pp. A2917-A2945, 2012.

[45] Rethore, J., Tinnes, J.-P., Roux, S., Buffiere, J.-Y., and Hild, F., Extended three-dimensional digital image correlation (X3D-DIC), Comptes Rendus Mécanique, vol. 336, pp. 643-649, 2008.

[46] Roux, S. and Hild, F., Digital image mechanical identification (DIMI), Experimental Mechanics, vol. 48, pp. 495-508, 2008.

[47] Roux, S., Hild, F., and Berthaud, Y., Correlation image velocimetry: a spectral approach, Applied Optics, vol. 41, pp. 108-115, 2002.

[48] Roux, S., Hild, F., Viot, P., and Bernard, D., Three-dimensional image correlation from X-ray computed tomography of solid foam, Composites Part AApplied Science and Manufacturing, vol. 39, pp. 1253-1265, 2008.

[49] Serfling, R. J., Approximation Theorems of Mathematical Statistics, John Wiley and Sons, 1980.

[50] Soize, C., Non-gaussian positive-definite matrix-valued random fields for elliptic stochastic partial differential operators, Computer Methods in Applied Mechanics and Engineering, vol. 195, pp. 26-64, 2006.

[51] Soize, C., Tensor-valued random fields for meso-scale stochastic model of anisotropic elastic microstructure and probabilistic analysis of representative volume element size, Probabilistic Engineering Mechanics, vol. 23, pp. 307323, 2008.

[52] Soize, C., Identification of high-dimension polynomial chaos expansions with random coefficients for non-gaussian tensor-valued random fields using 
partial and limited experimental data, Computer Methods in Applied Mechanics and Engineering, vol. 199, pp. 2150-2164, 2010.

[53] Soize, C., A computational inverse method for identification of nonGaussian random fields using the Bayesian approach in very high dimension, Computer Methods in Applied Mechanics and Engineering, vol. 200, pp. 30833099, 2011.

[54] Soize, C., Stochastic Models of Uncertainties in Computational mechanics, Vol. 2 of Lecture Notes in Mechanics, American Society of Civil Engineers, Reston, 2012.

[55] Spall, J. C., Introduction to Stochastic Search and Optimization, John Wiley and Sons, Hoboken, New Jersey, 2003.

[56] Ta, Q. A., Clouteau, D., and Cottereau, R., Modeling of random anisotropic elastic media and impact on wave propagation, European Journal of Computational Mechanics, vol. 19, pp. 241-253, 2010.

[57] Vendroux, G. and Knauss, W. G., Submicron deformation field measurements: Part 1. developing a digital scanning tunnelling microscope, Experimental Mechanics, vol. 38, pp. 18-23, 1998.

[58] Vendroux, G. and Knauss, W. G., Submicron deformation field measurements: Part 2. improved digital image correlation, Experimental Mechanics, vol. 38, pp. 86-92, 1998.

[59] Walter, E. and Pronzato, L., Identification of Parametric Models from Experimental Data, Springer, 1997. 